\title{
Research on the optimization task scheduling algorithm for arithmetic unit in microcomputer
}

\author{
Yang Diansheng \\ Office of Academic Affairs, Ezhou Polytechnic, Ezhou Hubei 436000, China
}

Keywords: anti-collision; task scheduling; combination weighting method

\begin{abstract}
With the continuous development of computer technology, the quantity of arithmetic unit tasks in intelligent microcomputer is more, a large number of requests are added to one arithmetic unit at the same time, leading to shorter task scheduling process cycle becoming. The traditional task scheduling only base on time-sharing request method. With the increasing of scheduling tasks, when scheduling cycle is small enough, the scheduling can only be finished with queue waiting mode, thus the scheduling efficiency is reduced. Therefore, the arithmetic unit tasks optimization scheduling model in microcomputer based on combination weighting method is proposed, according to the characteristics of task scheduling to shape the task selection model, and transform the task selection problem into multiple attribute decision problem, through combination weighting method to calculate the weight of each attribute, and sequence the task waiting to be completed on the basis of the weight values, the task waiting to be completed of largest comprehensive attribute value is regarded as priority, according to the priority task selection method to shape task scheduling model facing to resource optimization, so as to complete optimal scheduling tasks.
\end{abstract}

\section{Introduction}

The task scheduling efficiency of arithmetic unit in microcomputer, directly affects the performance of microcomputer arithmetic unit. Therefore, the task scheduling method of arithmetic unit in microcomputer, has always been a difficult problem being researched in computer management field, and is the hot subject currently [1]. At present, the mainstream of arithmetic unit task scheduling methods in microcomputer are arithmetic unit task scheduling method based on conflict tree [2], the arithmetic unit task scheduling method based on distributed decision algorithm [3] and the arithmetic unit task scheduling method based on neural network algorithm [4]. Among them, the most commonly used is the arithmetic unit task scheduling method based on neural network algorithm [5]. Since the arithmetic unit task scheduling methods in microcomputer has an irreplaceable role in the computer field, it has attracted more and more attention of the experts [6-8]. During the microcomputer arithmetic unit task scheduling process, assuming the quantity of arithmetic unit tasks in intelligent microcomputer is large, a large number of requests are added to one arithmetic unit at the same time, leading to shorter task scheduling process cycle becoming. The traditional task scheduling only base on time-sharing request method. With the increasing of scheduling tasks, when scheduling cycle is small enough, the scheduling can only be finished with queue waiting mode, thus the scheduling efficiency is reduced.

In order to avoid the defects of the traditional algorithm, the arithmetic unit tasks optimization scheduling model in microcomputer based on combination weighting method is proposed, according to the characteristics of task scheduling to shape the task selection model, and transform the task selection problem into multiple attribute decision problem, through combination weighting method to calculate the weight of each attribute, and sequence the task waiting to be completed on the basis of the weight values, the task waiting to be completed of largest comprehensive attribute value is regarded as priority, according to the priority task selection method to shape task scheduling model facing to resource optimization, so as to complete optimal scheduling tasks. 


\section{Principle of the arithmetic unit task optimization scheduling method in microcomputer based on combination weighting method}

Selection of the arithmetic unit in microcomputer. Because the task of the arithmetic unit in microcomputer shows some new characteristics, the traditional task scheduling model has been unable to meet the requirements. Thus, this paper on the basis of characteristics exists in the operation of arithmetic unit in microcomputer to shape task selection model.

There are a lot of studies for attribute selection of arithmetic unit tasks in microcomputer, but each concern of the study is not identical. This paper on the basis of characteristics exists in the operation of arithmetic unit in microcomputer to judge the task properties. Generally speaking, arithmetic unit tasks in microcomputer has two prominent characteristics: diversity of tasks and limited resource of task computing. This paper divides the task attributes into three kinds, table 1 is used to describe.

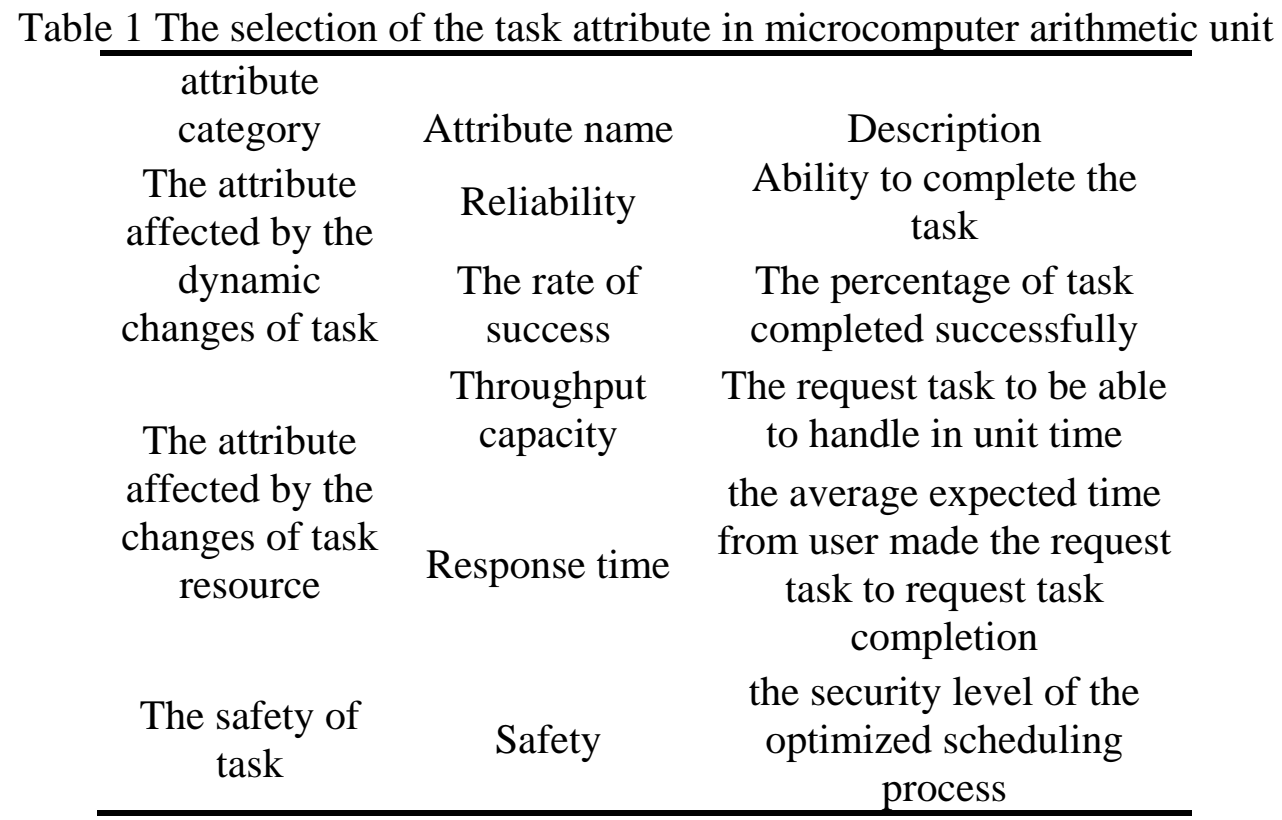

During the microcomputer arithmetic unit scheduling process, the task optimization scheduling model of multiple attribute types is a $n \times 4$ vector, $Q=\left\{\left(q_{1}, s_{1}, v_{1}, l_{1}\right),\left(q_{2}, s_{2}, v_{2}, l_{2}\right), \cdots,\left(q_{n}, s_{n}, v_{n}, l_{n}\right)\right\}$. Among them, $Q_{j}=\left(q_{j}, s_{j}, v_{j}, l_{j}\right)$ is used to describe the information of a certain attribute. $q_{j}$ is used to describe the name of the attribute, $s_{j}$ is used to describe the category of the properties belongs to, $v_{j}$ is used to describe the value of the attribute. If the attribute is different, the dimension and unit of it are different, thus, the dimensionless operation have to be completed for each attribute index;

If it is a single attribute, that is:

If it is a composite attribute, is:

$$
v_{j}=\left\{\begin{array}{c}
\frac{v_{j \text { max }}-v_{j \text { now }}}{v_{j \max }-v_{j \min }}, v_{j \text { max }}-v_{j \text { min }} \neq 0 \\
1,, v_{j \text { max }}-v_{j \text { min }}=0
\end{array}\right.
$$

$$
v_{j}=\left\{\begin{array}{c}
\frac{v_{j n o w}-v_{j \min }}{v_{j \max }-v_{j \min }}, v_{j \max }-v_{j \min } \neq 0 \\
1,, v_{j \text { max }}-v_{j \text { min }}=0
\end{array}\right.
$$

The optimal task scheduling based on combination weighting method. The comprehensive attribute value of arithmetic unit tasks in microcomputer is directly affected by the weight of each attribute, but when there are a large number of task attributes, if the value of attribute weights are 
only assigned by the user subjective, the accuracy and validity of decision will be poor. In order to avoid these problems, this paper will transform task selection problem into multiple attribute decision making problems, through combination weighting method to calculate the weight of each attribute, and ultimately achieve the goal that taking into account of the user's subjectivity and attribute data's objectivity at the same time.

Assuming that $W=\left\{W_{1}, W_{2}, \cdots, W_{m}\right\}$ is used to describe a collection of tasks need to be finished of an abstract task node, the $Q^{\prime}=\left\{q_{1}, q_{2}, \cdots, q_{n}\right\}$ is used to describe the arithmetic unit task attribute collection in microcomputer. Among them, $q_{j}$ is used to describe the name of the attribute, the attribute value of task $W_{i}$ to attribute $q_{j}$ can be expressed as $q_{i j}$, then, matrix $A=\left(q_{i j}\right)_{m \times n}$ is decision matrix. Among them, $i=1,2, \cdots, m ; j=1,2, \cdots, n$. On the basis of the above formula to normalize $A$, so as to obtain decision matrix $B=\left(p_{i j}\right)_{m \times n}$.

Supposing the subjective attribute weight vector input by the user input is $R=\left(R_{1}, R_{2}, \cdots, R_{n}\right)^{T}, 0 \leq R_{j} \leq 1, \sum_{j=1}^{n} R_{j}=1$. Therefore, the objective attribute weight vector is able to be obtained through the entropy method $r, r=\left(r_{1}, r_{2}, \cdots, r_{n}\right)^{T}, 0 \leq r_{j} \leq 1, \sum_{j=1}^{n} r_{j}=1$.

The combination of subjective and objective weight vector can help to obtain the combination weight $w=\alpha R+\beta r, \alpha+\beta=1$. In order to facilitate the analysis and discussion, let $\alpha=\beta=1 / 2$, then the combination weight of attribute $q_{j}$ of task $W_{i}$ can be expressed as $w_{i j}=\left(R_{i j}+r_{i j}\right) / 2$. Through weighted criteria of attribute decision studying to obtain comprehensive task attribute value:

$$
V_{i}=\sum_{j=1}^{n} p_{i j} w_{i j}=\sum_{j=1}^{n} p_{i j}\left(\alpha R_{i j}+\beta r_{i j}\right)=\frac{1}{2} \sum_{j=1}^{n}\left(R_{i j}+r_{i j}\right) p_{i j}
$$

Among them, $i=1,2, \cdots, m ; j=1,2, \cdots, n$.

The microcomputer arithmetic unit task selection is based on comprehensive attribute value to choose the task need to be executed in priority from available task collection need to be completed corresponding to each abstract task node. This paper on the basis of combination weighting method to get the comprehensive attribute of the task to compute the formula above, through the formula to calculate the comprehensive attributes for each task. In addition, the task need to be completed is sorted according to the comprehensive value, give priority to the task need to be completed of the largest comprehensive attribute value.

\section{Simulation results and analysis}

In order to verify the effectiveness of improved algorithms, there is the need for the experiment. By using the simulation software MATLAB 7.1 to construct the experiment environment. The experimental data is from real data in microcomputer arithmetic unit running process. Using the traditional algorithm and the improved algorithm to do contrast experiment.

First, analyzing the total number of microcomputer arithmetic unit tasks. The total number of scheduling is $T$, the total number of tasks is $N$. In the process of simulation, experiments for the number of tasks and scheduling times with slot time $\mathrm{k}=8, \mathrm{k}=16, \mathrm{~K}=24$ are processed respectively. The experimental results can be described as below: 


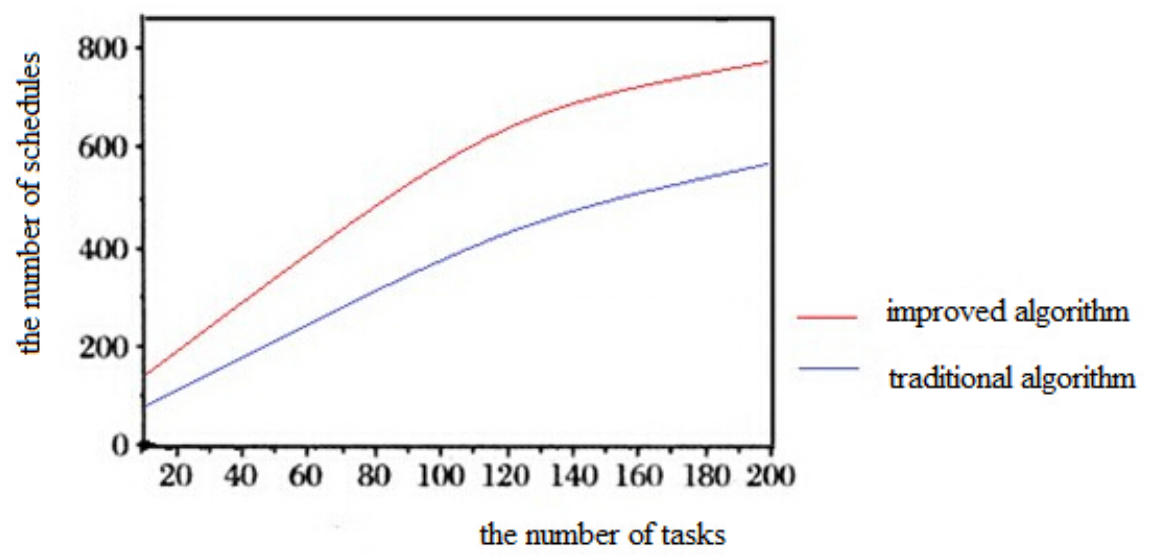

(a) $\mathrm{K}=8$

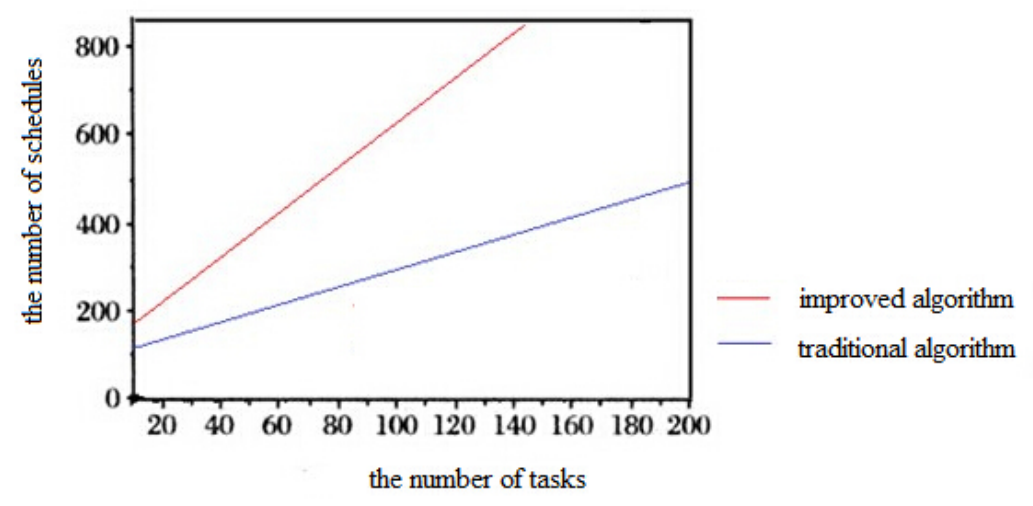

(b) $\mathrm{K}=16$

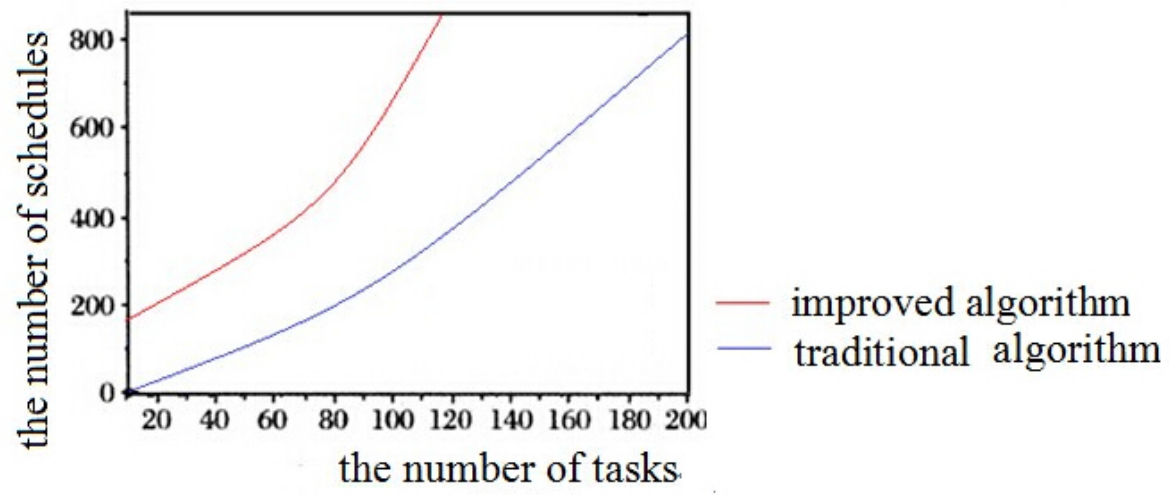

(c) $\mathrm{K}=24$

Fig. 1 Comparison of the scheduling number experimental results obtained with different algorithm

It can be learned from the above experimental results, the total number of scheduling of the improved algorithm is much higher than the total number of scheduling of the traditional algorithm.

The data obtained in the experimental process above is organized, the scheduling efficiency data of different algorithms are listed in the following table:

Table 2 experimental data of scheduling efficiency of different algorithms

\begin{tabular}{llll}
\hline Algorithm & $\begin{array}{l}\text { The number } \\
\text { experiments (times) }\end{array}$ & of & $\begin{array}{l}\text { The efficiency of } \\
\text { scheduling (\%) }\end{array}$ \\
\hline $\begin{array}{l}\text { Traditional } \\
\text { algorithm }\end{array}$ & 100 & 88 \\
$\begin{array}{l}\text { Improved } \\
\text { algorithm }\end{array}$ & 100 & 94 \\
\hline
\end{tabular}

According to the table above, it can be learnt that with the proposed algorithm to schedule microcomputer arithmetic unit tasks, can greatly reduce the probability of task conflict, and improve the scheduling efficiency, fully embodies the advantages of improved algorithm. 


\section{Conclusions}

In view of the defects of traditional algorithm for arithmetic unit task scheduling in the microcomputer, the arithmetic unit tasks optimization scheduling model in microcomputer based on combination weighting method is proposed, according to the characteristics of task scheduling to shape the task selection model, and transform the task selection problem into multiple attribute decision problem, through combination weighting method to calculate the weight of each attribute, and sequence the task waiting to be completed on the basis of the weight values, the task waiting to be completed of largest comprehensive attribute value is regarded as priority, according to the priority task selection method to shape task scheduling model facing to resource optimization, so as to complete optimal scheduling tasks.

\section{References}

[1] Gong Haigang, Liu Ming, Mao Yingchi, et al. Research progress of key technology for P2P large database data file [J]. Computer research and development, 2005, 42 (12): 2033-2040.

[2] Zhang Lifang. Research of data file scheduling strategy for P2P large database based on genetic algorithm [J]. Computer and digital engineering, 2009, 37 (4): 31-33.

[3] Sun Mingsong, Zhou Hongmin, Tang Liang. An adaptive data file scheduling algorithm for P2P large database [J]. Computer application, 2008, 28 (3): 558-567.

[4] Huang Shuqiang, Fu Zhongliang. An interference avoidance algorithm for wireless Mesh network channel assignment [J]. Journal of Wuhan University: Information Science Edition, 2013, (2):248-251.

[5] Wu Liming, Chen Taiwei, Xiang Ying. A new anti-collision algorithm of RFID sensor system in IOT [J]. Computer application, 2013, 33 (3):614-617.

[6] Abdelhalim M B, Habib S E-D. An integrated high-level hardware/software partitioning methodology [J].Des Autom Embed Sys, 2011, (01): 19-50.

[7] Xing J T, Xiong Y P, Price W G. Passive-Active Vibration Isolation Systems to Produce Zero or Infinite Dynamic Modulus: Theoretical and Conceptual Design Strategies [J]. Journal of Sound and Vibration, 2005.615-636.

[8] Ozdamar L. Emergency logistics planning in natural disasters [J]. Annal ofOperation Research, 2004, 129 (11): 218-219.

[9] Su Xiang, Zhu Li. Study of MPBP robust scheduling under uncertain condition [J]. Value engineering, 2012, 31 (24):200-202.

[10] Gao Li, Xu Kelin, Mao Daoxiao, Li Yanbing. Many varieties of order scheduling method of based on rule and polychromatic sets [J]. Journal of Tongji University: Natural Science Edition, 2013 (2): 258-263.

[11] Ma Xiaoting, liddy. Cloud computing environment based on load balancing of the digital library, virtual machine cluster resource scheduling strategy [J]. Modern intelligence, 2013, (3) : 3-5.

[12] Peng-wei li, wen-ying ge. Cloud computing environment to study the dynamic deployed virtual machine [J]. Computer measurement and control, 2013, 21 (5) : 1374-1376.

[13] QingJuan. Application of improved particle swarm algorithm in cloud computing application of task scheduling and simulation study [J]. Computer CD software and applications, 2014, (6) : 28-32.

[14] Fan Huali Xiong He root, Qian Guojie guo-zhang jiang, gong-fa li. Dynamic Job Shop scheduling problems setting up the delivery time of the simulation of research [J]. Journal of manufacturing automation, 2014, 4 (8) : 66-68.

[15] Ya-dong liu, HongBing Chen Xiangbin, xue-feng gu, liu. Based on dynamic programming algorithm of equipment support tasks scheduling priority [J]. Journal of military traffic institute, 2014 (4) : 24-29. 